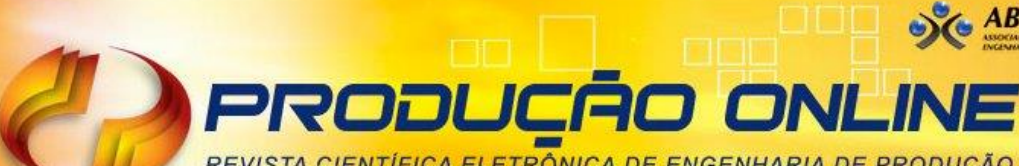 REVISTA CIENTIFICA ELETRÔNICA DE ENGENHARIA DE PRODUÇÃO

\section{APLICAÇÃO DE ALGORíTMO GENÉTICO E DA METHAEURÍSTICA SIMULATED ANNELING EM UM PROCESSO SIDERÚRGICO}

\section{APPLICATION OF GENETIC ALGORITHM AND SIMULATED ANNELING METHAEURISTICS IN A STEEL PROCESS}

\author{
Cristie Diego Pimenta*E-mail: pimentadiego@yahoo.com.br \\ Messias Borges Silva* E-mail: messias.silva@feg.unesp.br \\ Fernando Augusto Silva Marins* E-mail: fernando.marins@outlook.com \\ *Universidade Estadual Paulista "Júlio de Mesquita Filho" (UNESP), São Paulo, SP, Brasil
}

\begin{abstract}
Resumo: O objetivo deste artigo é mostrar a aplicação de algoritmo Genético e de Simulated Anneling para otimizar as predições estatísticas para o processo de tratamento térmico de têmpera em arames de aço. Essa modelagem estatística pode ser capaz de substituir o processo utilizado para a preparação de fornos de têmpera e revenimento, que tradicionalmente é realizada por meio de ajustes feitos a partir do resultado da propriedade mecânica dureza, ensaiada em laboratório e exigida para atender as especificações de clientes. Buscou-se compreender a influência das variáveis de entrada (fatores) e os seus efeitos na propriedade mecânica dureza, em arames de aço SAE 9254, para o diâmetro 2,00 mm, utilizado para a fabricação de molas de válvula e de embreagem para o seguimento automobilístico. Foram investigadas as principais variáveis de entrada do processo e para isso, utilizaram-se as metodologias Regressão Múltipla Quadrática e a Metodologia de Superfícies de Resposta (RSM). Para a otimização do modelo estatístico foram utilizadas as metodologias Algoritmo Genético (AG) e a Meta-heurística Recozimento Simulado (Simulated Anneling). Os resultados revelaram que é possível se obter bons resultados se utilizada essa modelagem estatística e se o modelo estatístico for otimizado por meio das técnicas aplicadas neste artigo. Se as metodologias forem aplicadas corretamente, isso poderá trazer avanços científicos que poderiam proporcionar a automatização deste processo, e consequentemente isso poderia contribuir para o aumento de produtividade e da qualidade do produto.
\end{abstract}

Palavras-chave: Tratamento Térmico. SAE 9254. Metodologia de Superfície de Resposta. Algorítmos Genéticos. Meta-heurística.

Abstract: The aim of this article is to show the application of Genetic and Simulated Anneling algorithms to optimize statistical predictions for the process of tempering heat treatment in steel wires. This statistical modeling may be able to replace the process used for the preparation of tempering and tempering furnaces, which is traditionally carried out by means of adjustments made from the result of the mechanical hardness property, tested in the laboratory and required to meet customer specifications. We sought to understand the influence of the input variables (factors) and their effects on the mechanical property hardness, in SAE 9254 steel wires, for the $2.00 \mathrm{~mm}$ diameter, used for the manufacture of valve and clutch springs for the follow-up automobile. The main input variables of the process were investigated and for that, the Quadratic Multiple Regression and the Response Surface Methodology (RSM) were used. For the optimization of the statistical model, the methodologies Genetic Algorithm (AG) and the Simulated Annealing Meta-heuristic were used. The results revealed that it is possible to obtain good results if this statistical model is used and if the statistical model is optimized through the techniques applied in this article. If the methodologies are applied correctly, this could bring scientific advances that could provide the automation of this process, and consequently this could contribute to the increase in productivity and product quality. 
Palavras-chave: Heat Treatment. SAE 9254. Response Surface Metodology, Genetic algorithms. Meta-heuristic.

\section{INTRODUÇÃo}

Nesse artigo, será apresentada a aplicação das metodologias de Superfícir de Resposta, Algoritmo Genético e Simulated Anneling para a criação de uma modelagem estatística que possa substituir a maneira tradicional de erro - tentativa no ajuste das variáveis de entrada do forno de tratamento térmico. No processo em questão, o ajuste inicial (setup) é realizado por meio do ensaio da propriedade mecânica dureza em uma amostra-piloto que, após passar por todas as fases de um tratamento térmico de têmpera e revenimento, é encaminhada para a análise em laboratório.

O resultado da dureza obtido, nessa etapa, é usado para configurar a regulagem do forno dentro da qual se faz uma segunda amostra-piloto, para confirmar que os ajustes do processo foram suficientes para que o produto venha a atingir a especificação mecânica. Essa rotina operacional implica em considerável tempo de análise e de espera, reduzindo a produtividade do processo devido ao baixo rendimento, visto que o forno permanece inoperante até que seja configurado.

\section{REVISÃO DA LITERATURA}

\subsection{Processo de têmpera e revenimento}

Segundo Callister (2012), a têmpera está relacionada ao resfriamento brusco do aço após aquecimento à temperatura de austenitização e tem como objetivo a obtenção de uma microestrutura que proporcione propriedades mecânicas, tais como dureza e limite de resistência elevadas para aplicações específicas que requerem essa condição. Durante o estágio de resfriamento na têmpera a queda de temperatura promove transformações estruturais que acarretam o surgimento de tensões internas e por isso se faz necessária a realização do revenimento, quando ocorre a transformação da martensita em martensita revenida.

O revenido envolve uma série de transformações micro estruturais que tendem ao equilíbrio termodinâmico. É, portanto, um processo termicamente ativado e, assim, função direta do tempo e da temperatura de processo (PINTO, 2003). 
O processo de revenimento é realizado complementarmente à têmpera sendo especialmente importante na fabricação de aços para molas. Ele consiste em aquecer o material temperado entre $250^{\circ} \mathrm{C}$ a $650^{\circ} \mathrm{C}$ por um determinado tempo, para aumentar a ductilidade e elasticidade (CALLISTER, 2012).

\subsection{Ensaios de dureza}

Segundo Chiaverini (2012), a dureza é a medida da resistência de um metal à penetração. Os métodos mais comuns de se determinar a dureza de um metal são o Brinell, o Vickers e o Rockwell. Neste trabalho somente será utilizado o método Brinell (HB).

As propriedades mecânicas dureza e limite de resistência à tração estão diretamente relacionadas. Em geral quanto maior a dureza, maior será o limite de resistência à tração e como frequentemente a dureza é proporcional à resistência ao desgaste e durabilidade nos aços, ela é utilizada como uma forma de medir a resistência à abrasão.

A obtenção dos valores de dureza Brinell $(\mathrm{HB})$, conforme mostrado na Figura 1, é feita dividindo-se a carga aplicada pela área de penetração. O penetrador de diâmetro (D) é uma esfera de aço temperado para materiais de dureza média ou baixa, ou de carboneto de tungstênio, para materiais de elevada dureza. A máquina de ensaio possui um microscópio óptico que faz a medição do diâmetro do círculo $(d$, em $\mathrm{mm})$ que corresponde à projeção da calota esférica impressa na amostra. A dureza Brinell $(\mathrm{HB})$ será dada pela carga aplicada $(P$, em kgf) dividida pela área de impressão, conforme (1):

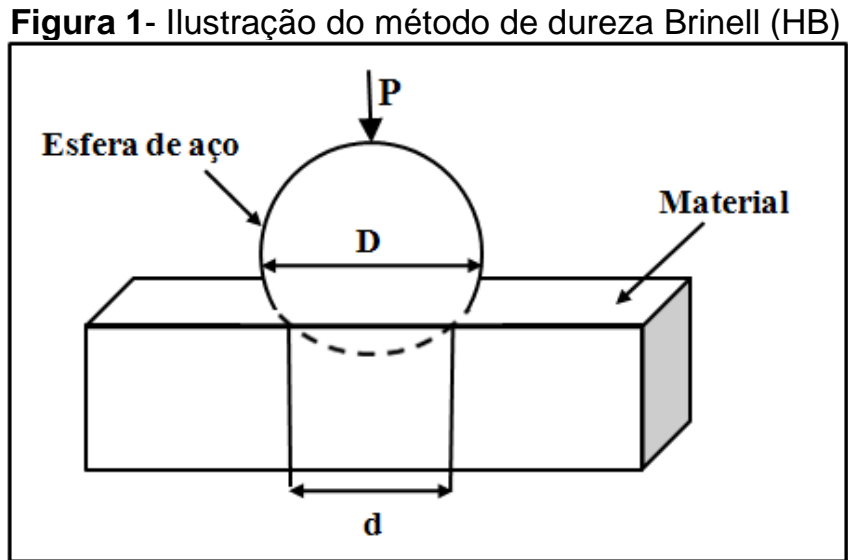




$$
H B=\frac{2 P}{\pi D\left(D-\sqrt{D^{2}}-d^{2}\right)} \quad\left[\mathrm{kgf} / \mathrm{mm}^{2}\right]
$$

\subsection{Métodos estatísticos utilizados}

Segundo Teófilo et.al (2004) a Metodologia da superfície de resposta (Response Surface Methodology- RSM) baseia-se na construção de modelos matemáticos empíricos que geralmente utiliza-se de funções polinomiais quadráticas para oferecer condições de otimização para sistemas.

A Metodologia de superfícies de resposta ou RSM é uma técnica estatística para modelar processos e otimizar as variáveis resposta, buscando um máximo ou um mínimo de rendimento no qual se deseja otimizar. Normalmente, se utiliza um planejamento fatorial $2^{2}$, investigando dois fatores, para investigação da região ótima, por meio do caminho de ascensão máximo ou mínimo, dependendo do que se deseja otimizar e o Método dos Mínimos Quadrados é utilizado para estimar os parâmetros do modelo quadrático (polinomial), utilizado para encontrar o melhor ajuste dos fatores, que proporcionará atingir a melhor variável resposta (SILVA, 2013).

Segundo Neto et al. (2007), a Metodologia de superfícies de resposta (ou RSM, de Response Surface Methodology) é uma técnica de otimização baseada em planejamentos fatoriais e tem sido usada com grande sucesso na modelagem de diversos tipos de processos industriais. Para aplicação da Metodologia de superfícies de resposta é necessário duas fases, modelagem e deslocamento. Que deverão ser realizadas tantas vezes se fizer necessário, até que se obtenha a região ótima da superfície investigada. Essa modelagem é realizada por meio do ajuste de modelos lineares ou quadráticos, nas quais as variáveis respostas são obtidas por meio da execução de planejamentos fatoriais. O deslocamento é feito por meio do caminho de ascensão máxima de inclinação de um determinado modelo, que é a trajetória na qual a variável resposta varia, podendo ocasionar curvatura, que ocorre quando uma determinada resposta é aumentada gradativamente até que em um determinado ponto, começa a sofrer queda, esse é chamado ponto de curvatura da resposta. 
Conforme Mark e Shari (2003) e Bajic et.al (2010) a Metodologia de superfícies de resposta (RSM) pode ser aplicada, posteriormente, não somente aos experimentos executados por Delineamento de experimentos (DOE), mas também a partir de aplicações de matrizes do Método de Taguchi, sendo uma técnica essencial e eficaz utilizada para modelar matematicamente situações em que o modelo linear (1 ${ }^{a}$ ordem) não satisfaz e há necessidade de uma modelagem polinomial ( $2^{a}$ ordem) para representar adequadamente o processo.

Frequentemente as condições iniciais planejadas e executadas por Delineamento de Experimentos estão afastadas daquelas condições que otimizam a resposta. Em tais condições, utilizando a Metodologia de superfícies de resposta (RSM), primeiramente o objetivo é mover o experimento para a região ótima, utilizando o caminho de ascensão máximo ou mínimo de inclinação, também chamados de inclinação ascendente (steepest ascent) ou máxima inclinação descendente (steepest descent), que é um procedimento experimental que busca a melhor região para a resposta, seja para aumento da resposta ou redução (PAIVA, 2006).

É mostrado na Figura 2, o gráfico de contornos dos valores preditos da variável resposta, e o caminho de ascensão máxima, que é uma série de linhas paralelas. Após se encontrar a região ótima para a variável resposta, se faz necessário o planejamento estrela, que dará origem à função quadrática para modelagem do processo.

O planejamento estrela é realizado por meio de duas combinações de tratamento axial nos níveis alto e baixo para cada fator, mantendo os demais fixos, nos pontos centrais, acrescidos de um ponto unitário no ponto central para todos os outros fatores (TEÓFILO et.al, 2004).

Figura 2 - Caminho de ascensão máxima de inclinação

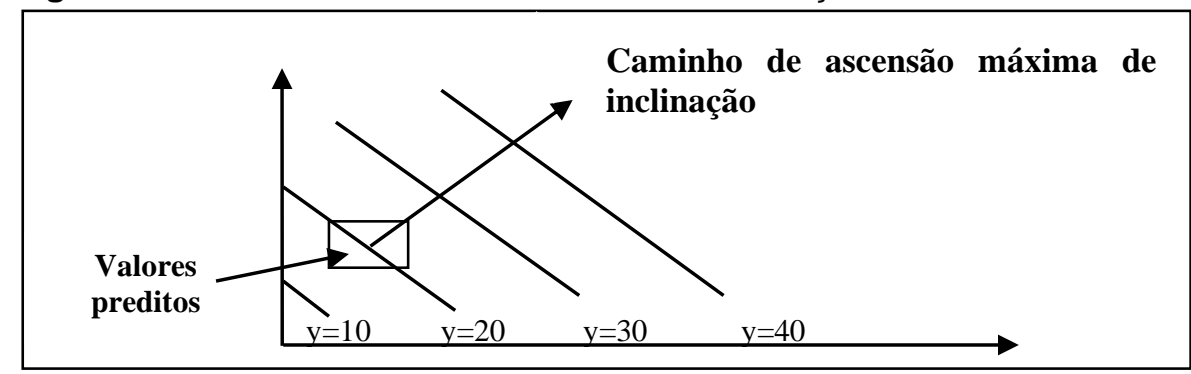


Os termos quadráticos e de interação alcançados por meio da Metodologia de Superfícies de Resposta, podem produzir uma função formando uma curvatura, chamado modelo de $2^{\mathrm{a}}$ ordem. Dependendo dos valores dos coeficientes de regressão, o modelo de segunda ordem com interação é capaz de considerar uma ampla variedade de formas (MONTGOMERY; RUNGER, 2003).

Como a maioria dos problemas que envolvem a superfície de resposta é desconhecida, o primeiro procedimento é encontrar uma aproximação para o verdadeiro relacionamento entre a variável resposta $(y)$ e as variáveis independentes (fatores) e geralmente utiliza-se uma modelagem matemática por meio de uma regressão polinomial (quadrática). A seguir, é apresentado esse modelo de regressão polinomial de segunda ordem (2):

$$
y=\beta_{0}+\sum_{i=1}^{k} \beta_{i} x_{i}+\sum_{i=1}^{k} \beta_{i i} x_{i}^{2}+\sum_{i=1}^{k-1} \sum_{j>i}^{k} \beta_{i j} x_{i} x_{j}+\varepsilon
$$

Sendo:

ßo representa a média da variável resposta;

$\sum_{i=1}^{k} \beta_{i} x_{i} \quad$ representa o efeito linear;

$\sum_{i=1}^{k} \beta_{i i} x_{i}^{2} \quad$ representa o efeito quadrático;

$\sum_{i=1}^{k-1} \sum_{j>i}^{k} \beta_{i j} x_{i} x_{j}+\varepsilon$ representa o efeito da interação.

Segundo Rodrigues et al. (2004), inspirada pela Seleção Natural de Darwin em 1859, a Computação Evolucionária (CE) é um ramo de pesquisa emergente da Inteligência Artificial que propõe um novo paradigma para solução de problemas. Com isso, a Computação Evolucionária compreende um conjunto de técnicas de busca e otimização que cria uma população de indivíduos que vão reproduzir e competir pela sobrevivência. Atualmente, as técnicas de Computação Evolucionária incluem: Programação Evolucionária, Estratégias Evolucionárias, Algoritmos Genéticos (concebidos em 1960 por John Holland) e Programação Genética. Estes métodos estão sendo utilizados, cada vez mais, pela comunidade de inteligência 
artificial para obter modelos de inteligência computacional (COSTA, 2006). Neste artigo, somente será tratado o assunto referente aos Algoritmos Genéticos (AG).

Os Algoritmos Genéticos (AGs) possuem uma larga aplicação em muitas áreas científicas, entre as quais podem ser citados problemas de otimização de soluções, aprendizado de máquina, desenvolvimento de estratégias e fórmulas matemáticas, análise de modelos econômicos, problemas de engenharia, diversas aplicações na Biologia como simulação de bactérias, sistemas imunológicos, ecossistemas, descoberta de formato e propriedades de moléculas orgânicas (ZINI, 2009).

Segundo Holland (1975), os indivíduos mais aptos terão um maior número de descendentes, ao contrário dos indivíduos menos aptos. Os requisitos para a implementação de um AG são:

a. Representações das possíveis soluções do problema no formato de um código genético;

b. População inicial que contenha diversidade suficiente para permitir ao algoritmo combinar características e produzir novas soluções;

c. Existência de um método para medir a qualidade de uma solução potencial;

d. Um procedimento de combinação de soluções para gerar novos indivíduos na população;

e. Um critério de escolha das soluções que permanecerão na população ou que serão retirados desta;

f. Um procedimento para introduzir periodicamente alterações em algumas soluções da população. Desse modo mantém-se a diversidade da população e a possibilidade de se produzir soluções inovadoras para serem avaliadas pelo critério de seleção dos mais aptos.

A ideia básica de funcionamento dos Algoritmos genéticos (AGs) é a de tratar as possíveis soluções do problema como "indivíduos" de uma "população", que irá "evoluir" a cada iteração ou "geração". Para isso é necessário construir um modelo de evolução nos quais os indivíduos sejam soluções de um problema (HOLLAN, 1975). Segundo Holland (1975), a execução do algoritmo pode ser resumida nos seguintes passos: 
g. Escolher uma população inicial, formada por indivíduos criados aleatoriamente;

h. Avaliar toda a população de indivíduos segundo algum critério, determinado por uma função que avalia a qualidade do indivíduo (função de aptidão);

i. Por meio do operador de seleção, escolhem-se os indivíduos de melhor valor (dado pela função de aptidão) como base para a criação de um novo conjunto de possíveis soluções, chamado de nova "geração";

j. Esta nova geração é obtida aplicando-se sobre os indivíduos selecionados operações que misturem suas características (chamadas "genes"), por meio dos operadores de "cruzamento") e "mutação";

k. k.Estes passos são repetidos até que uma solução aceitável seja encontrada, até que o número predeterminado de passos seja atingido ou até que o algoritmo não consiga mais melhorar a solução já encontrada.

O principio básico dos operadores genéticos é transformar a população por meio de sucessivas gerações, estendendo a busca até chegar a um resultado satisfatório. Os operadores genéticos são necessários para que a população se diversifique e mantenha as características de adaptação adquiridas pelas gerações anteriores. Por meio do cruzamento são criados novos indivíduos, misturando características de dois indivíduos "pais". Esta mistura é feita tentando imitar a reprodução de genes em células e o resultado desta operação é um indivíduo que potencialmente combine as melhores características dos indivíduos usados como base (HOLLAN, 1975).

Os Algoritmos Genéticos (AGs) são métodos robustos e têm sido apontados como técnicas promissoras para solucionar problemas combinatórias tais como, os problemas de programação inteira, pois possuem uma reconhecida habilidade em atingir soluções próximas ao ótimo em problemas de larga escala (COSTA, 2006).

Segundo Zini (2009), as principais vantagens que podem ser consideradas na utilização dos Algoritmos Genéticos são:

a. São robustos e aplicáveis a uma grande variedade de problemas;

b. Não usam apenas informação local, logo, não ficam presos, necessariamente, a ótimos locais como determinados métodos de busca. Esta característica é 
uma das mais interessantes dos algoritmos genéticos e fazem com que eles sejam técnicas extremamente adequadas para funções multimodais e de comportamento complexo;

c. Seu desempenho não é afetado por descontinuidades na função ou em suas derivadas. Os algoritmos genéticos não usam informações de derivadas na sua evolução nem necessitam de informação dos gradientes da superfície da função objetivo para efetuar a busca. Isto faz com que sejam muito adequados para funções com descontinuidades ou para os quais não temos como calcular a derivada;

d. Apresentam um bom desempenho para uma grande escala de problemas;

e. São de fácil implementação e proporcionam maior flexibilidade no tratamento do problema a ser resolvido;

Segundo Zini (2009), as desvantagens dos Algoritmos Genéticos são:

a. Dificuldade de achar o ótimo global exato;

b. Requerem um grande número de avaliações de função de aptidão;

c. Grandes possibilidades de configurações que podem complicar a resolução do problema tratado.

O problema de otimização global de funções numéricas tem grande importância em várias áreas do conhecimento, surgindo nas áreas da Engenharia, economia, entre outras (CHERRUAULT, 2005). A função a ser otimizada aparece como um índice de performance multivariável e está sujeita a certos vínculos, impostos por restrições de processo (JUNIOR, 2008).

Durante as últimas décadas, heurísticas clássicas foram desenvolvidas em diferentes estágios, sendo que na década de 80 tornaram-se as proposições mais populares para solução de problemas práticos. Devido aos grandes avanços científicos dos últimos anos, as heurísticas foram revistas e aperfeiçoadas, resultando na nova classe de heurísticas chamadas meta-heurísticas (OLIVEIRA, 2004).

As técnicas meta-heurísticas são ferramentas fundamentais para resolução de problemas de otimização complexos cujos espaços de busca das soluções ótimas sejam muito grandes para que se possa determiná-las com precisão por meio de um método determinístico com tempo de processamento aceitável (CHAVES et. Al, 2007). 
Nos métodos heurísticos de melhoria, obtém se uma solução inicial e posteriormente por meio de algum procedimento interativo (geralmente envolvendo trocas de posições das tarefas na sequência) busca-se obter uma sequência das tarefas melhor que a atual quanto à medida de desempenho adotada. Para isso, foi desenvolvido o método de busca em vizinhança de maior complexidade (Simulated Annealing) que têm sido alvo de grande interesse na comunidade científica em função de aplicações bem sucedidas e portadas na literatura (BUZZO; MOCELLIN, 2000).

Simulated Annealing, Têmpera Simulada ou Recozimento Simulado é uma técnica de busca local que simula o processo de recozimento de metais, no qual o metal é aquecido a altas temperaturas e, em seguida, é feito um resfriamento sistemático do mesmo visando atingir um ponto de equilíbrio caracterizado por uma microestrutura ordenada e estável (LIBRANTZ, 2010).

Segundo Diógenes (2009), o algoritmo do Recozimento Simulado, deriva da observação de que a solução de problemas combinatórias de larga escala é análoga ao recozimento de sólidos no campo da física da matéria condensada. O objetivo deste processo é reduzir a temperatura de um sistema, levando-o ao estado de mínima energia. Tal energia pode ser vista como uma função custo a ser otimizada.

A Meta-heurística Recozimento Simulado é uma meta-heurística proposta por Kirkpatrick et al. (1983), sendo uma técnica de busca probabilística que se fundamenta em uma analogia com a termodinâmica, ao simular o resfriamento de um conjunto de átomos aquecidos.

Sua origem está relacionada ao ajuste das propriedades mecânicas por meio de um processo de resfriamento controlado, no qual o produto é aquecido à determinada temperatura e, então, resfriado em escalas de resfriamento, de acordo com o resultado desejado. Se o objetivo é obter dureza e rigidez, a temperatura é diminuída abruptamente. Se, ao contrário, desejamos flexibilidade, a redução é feita lentamente, até a temperatura ambiente (JUNIOR, 2008). O pseudocódigo do algoritmo é apresentado na Figura 3.

Conforme Barros e Moccellin (2004), o uso da Meta-heurística Recozimento Simulado é justificado pela capacidade de efetuar movimentos "colina acima" no espaço de soluções viáveis do problema, explorando os "vales" na tentativa de se obter uma solução ótima global para o problema. O Recozimento Simulado pode ser 
considerado uma generalização do método "descendente", no qual a busca não estendida para um mínimo global é terminada após um mínimo local ser obtido, podendo ser classificado como método heurístico de busca aleatória na vizinhança.

Figura 3 - Algoritmo Recozimento Simulado.

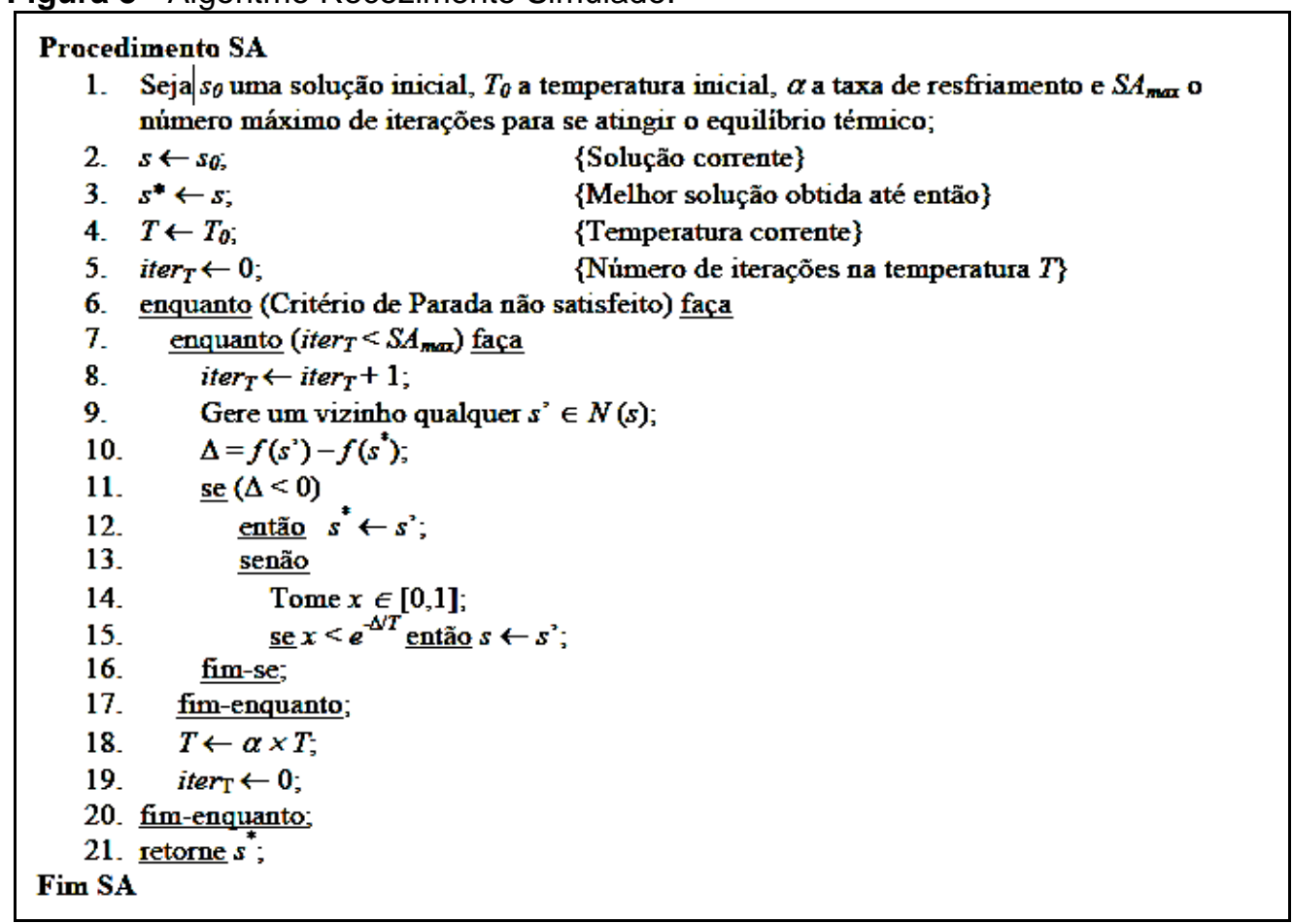

Fonte: Toffolo et al. (2005).

Segundo Ferreira et al. (2008) o Recozimento Simulado começa sua busca a partir de uma solução inicial qualquer. O procedimento principal consiste em um loop que gera aleatoriamente, em cada interação, um único vizinho s'da solução corrente s. Se este vizinho for melhor que o original, ele é aceito e substitui a solução corrente. Caso contrário, ele pode ser aceito com uma probabilidade e-D/T (no caso de problemas de minimização, ou eD/T, no caso de problemas de maximização) sendo $T$, um parâmetro chamado de Temperatura, que decresce gradualmente conforme o progresso do algoritmo. Esse processo é repetido até que $T$ seja tão pequeno que mais nenhum movimento de piora seja aceito. A melhor solução encontrada durante a busca é tomada como uma aproximação para a solução ótima (SOUZA, 2007). 
A meta-heurística Recozimento Simulado permite a mudança de uma solução para outra na vizinhança. $O$ valor de $T$ decresce gradativamente atingindo um valor próximo de zero, de modo que a troca de uma solução atual só é permitida quando for encontrada uma solução vizinha com desempenho superior (BUZZO, 2005).

A aplicação do Recozimento Simulado para a solução do problema de otimização requer a tomada de decisões específicas para cada problema, como a determinação de métodos de geração da solução inicial; a definição da vizinhança a ser considerada; a porcentagem da vizinhança analisada; a temperatura inicial; a condição de parada, entre outras (BUZZO, 2005).

Segundo Yamamoto (2004), uma característica importante do Recozimento Simulado é a aceitação de configurações que apresentam maior energia, que pode parecer pior, porém permite que o método não convirja para um mínimo local, podendo convergir para um melhor resultado.

\section{MATERIAIS}

O material usado neste estudo foi o aço SAE 9254 trefilado a frio, utilizado para fabricação de molas de válvulas e de embreagem aplicadas ao segmento automobilístico, com diâmetro de $2,00 \mathrm{~mm}$, submetido ao processo de têmpera e revenimento.

A análise química do material SAE 9254 usado no estudo é apresentada na Tabela 1.

Tabela 1 - Composição química (SAE 9254).

\begin{tabular}{cccccccccccc}
\hline $\begin{array}{l}\text { Elementos } \\
\text { Químicos }\end{array}$ & $\mathbf{C}$ & $\mathbf{M n}$ & $\mathbf{S i}$ & $\mathbf{P}$ & $\mathbf{S}$ & $\mathbf{C r}$ & $\mathbf{N i}$ & $\mathbf{M o}$ & $\mathbf{C u}$ & $\mathbf{A l}$ & $\mathbf{V}$ \\
\hline$(\%)$ & 0,554 & 0,64 & 1,22 & 0,022 & 0,018 & 0,58 & 0,04 & 0,03 & 0,01 & 0,009 & 0,005 \\
\hline
\end{tabular}

\section{APLICAÇÃO DA METODOLOGIA DE SUPERFÍCIES DE RESPOSTA}

Estudos preliminares comprovaram que a propriedade mecânica dureza possui curvatura influente, indicando que em um determinado ajuste dos parâmetros (fatores) ocorrerá uma curvatura na variável resposta. Existe a evidência de que ao se aumentar o valor de velocidade (representada pela letra A) e baixar a temperatura do chumbo (representada pela letra B), a dureza será aumentada, porém, as evidências apontam para que em um determinado ajuste dos fatores essa 
propriedade mecânica atinja o seu ponto máximo e em seguida, comece a decair, provocando então a curvatura da variável resposta dureza. Esse aumento do valor da dureza, proveniente do aumento do fator (A) poderá ser constatado pela observação prática, pois quanto mais a velocidade é aumentada teoricamente se amplia o efeito do choque-térmico no momento da têmpera, na qual o arame de aço após passar pelas fases de aquecimento do forno, entra em contato com o resfriamento em polímero mais rapidamente, e isso significaria um arame mais aquecido na hora do resfriamento, pois com uma alta velocidade não haveria tempo para dissipação de calor do arame na atmosfera. Porém, existe um limite máximo para o aumento dos valores da dureza, em função de (A), pois ao se aumentar excessivamente, ultrapassando o ponto (chamado curvatura), o arame passará tão rápido pela têmpera que não haverá tempo suficiente para que ocorra a transformação micro estrutural, ou seja, com excesso de velocidade, a mudança de estrutura bruta (perlita+ferrita) para martensita revenida, não ocorre, pois a velocidade está diretamente relacionada ao tempo de exposição do arame à têmpera e revenimento, e acima do ponto de curvatura, o tempo de exposição seria insuficiente para essa transformação micro estrutural.

Para a realização do planejamento de experimentos, foram utilizadas variáveis padronizadas $(\beta)$ ao invés de variáveis físicas (ajustes reais) dos fatores investigados, de forma a se preservar os dados confidenciais da empresa financiadora da pesquisa. A padronização das variáveis foi calculada segundo Montgomery e Runger (2003), utilizando o valor físico ( $\alpha$ ) que se quer testar subtraído da média $(\mu)$ entre os valores mínimo e máximo dos ajustes dos fatores. $O$ resultado dividiu-se pela metade da amplitude $(R)$ entre os valores mínimos e máximos dos ajustes dos fatores. Dessa forma, a dimensionalidade das variáveis padronizadas ficou restrita ao intervalo [-1 a 1]. Todavia, para a aplicação da Metodologia de superfície de resposta os valores irão extrapolar os limites estabelecidos de [-1 a 1], o que é normal para a aplicação desta metodologia. A fórmula para padronização de variáveis seguirá conforme (3):

$$
\beta=\frac{\alpha-\mu}{\frac{R}{2}}
$$




\subsection{Superfícies de Resposta para Dureza (HB)}

Primeiramente, para analisar a superfície de resposta da variável resposta dureza, foi realizado o chamado experimento estrela, que se refere a uma sequência de experimentos planejados na região a qual se encontra a curvatura, buscando uma modelagem quadrática do processo. Para o experimento estrela, foram estudados somente os dois principais fatores (A e B), pois juntos, explicam quase toda a influência gerada na resposta dureza. A sequência da experimentação (experimento estrela) e os resultados obtidos após execução dos experimentos são apresentados na Tabela 2.

Tabela 2 - Matriz experimental para modelagem quadrática da Dureza - HB

\begin{tabular}{cccccccc}
\hline $\begin{array}{c}\text { VELOCIDADE } \\
(\mathbf{A})\end{array}$ & $\begin{array}{c}\text { TEMPERATURA } \\
\text { (B) }\end{array}$ & \multicolumn{5}{c}{ DUREZA - Réplicas } \\
\hline-1 & -1 & 608 & 606 & 606 & 611 & 611 & 611 \\
1 & -1 & 608 & 608 & 608 & 608 & 608 & 608 \\
-1 & 1 & 544 & 542 & 542 & 542 & 542 & 542 \\
1 & 1 & 544 & 544 & 542 & 550 & 550 & 550 \\
$-1,41$ & 0 & 530 & 532 & 533 & 533 & 537 & 530 \\
1,41 & 0 & 550 & 553 & 554 & 548 & 551 & 556 \\
0 & $-1,41$ & 630 & 628 & 627 & 628 & 633 & 638 \\
0 & 1,41 & 517 & 520 & 516 & 520 & 522 & 519 \\
0 & 0 & 578 & 575 & 578 & 578 & 578 & 581 \\
\hline
\end{tabular}

Após a realização dos experimentos com a finalidade da modelagem quadrática, foi realizado um teste de significância (usando estatística Tstudent). Por meio do teste de significância (usando estatística Tstudent), apresentado na Tabela 3, foi possível constatar que os fatores $(A)$ e (B) são influentes, assim como, o termo quadrático $A$, com valores de $P$ inferiores a 0,05 (para $95 \%$ de confiança). Porém, foi observado que o termo $B^{2}$ e a interação não possuem influência significativa.

Tabela 3 - Teste de significância para modelagem quadrática da Dureza - HB

\begin{tabular}{cccc}
\hline Termos & Coeficiente & $\mathbf{T}$ & $\mathbf{p}$ \\
\hline Constante & 578,0 & 135,067 & 0,000 \\
(A) & 3,885 & 2,568 & 0,013 \\
(B) & $-35,719$ & $-23,608$ & 0,000 \\
$(\mathrm{~A})(\mathrm{A})$ & $-13,396$ & $-5,339$ & 0,000 \\
$(\mathrm{~B})(\mathrm{B})$ & 2,896 & 1,154 & 0,254 \\
$(\mathrm{~A})(\mathrm{B})$ & 1,292 & 0,604 & 0,549 \\
\hline
\end{tabular}


Analisando a Figura 4, é possível observar que a redução do fator (B) do nível 1 para -1 provoca aumento na dureza. Observou-se que o aumento do fator $(A)$ do estágio -1 para zero (0) provoca aumento na dureza. Porém, a dureza sofreu queda, quando o aumento foi acima do ponto zero (0), pois o tempo de processamento foi insuficiente para provocar a têmpera e revenimento no material, impossibilitando a transformação micro estrutural de estrutura bruta (perlita+ferrita) para martensita revenida. Com isso, achou-se o ponto máximo de velocidade admitida para obtenção do maior resultado de dureza.

Figura 4 - Superfície de resposta da Dureza - HB)

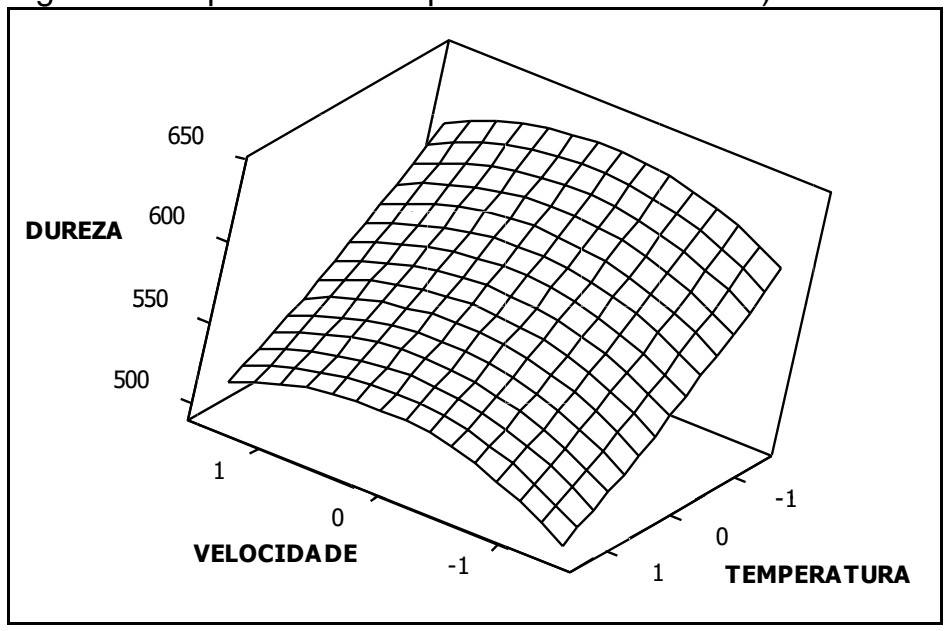

\subsection{Modelagem quadrática do processo de têmpera e revenimento}

Utilizando os coeficientes foi possível a criação do modelo quadrático que representa o processo, referente a propriedade mecânica dureza conforme (4):

Dureza $=578+3,885(A)-35,719(B)-13,396\left(A^{2}\right)$

\section{OTIMIZAÇÃO DO MODELO QUADRÁTICO}

Buscou-se a otimização do processo por meio da aplicação dos métodos Algoritmo Genético (AG) e da Meta-heurística Recozimento Simulado (Simulated Anneling). Para isso, foi utilizado o Software CrystalBall (para AG) e o Software Scilab (para Simulated Anneling). Nesta etapa, a otimização ocorrerá para os resultados obtidos de três clientes deste produto. Dessa forma, será possível testar 
o modelo (Quadrático), otimizando-o por dois métodos diferentes, em situações diferentes e podendo assim analisar os erros obtidos pelos diferentes métodos.

$\mathrm{Na}$ Tabela 4 é possível observar os valores especificados para a propriedade mecânica dureza.

Tabela 4 - Especificação da propriedade mecânica dureza (HB)

\begin{tabular}{cccc}
\hline & Mínina & Nominal & Máxima \\
Dureza $(\mathrm{HB})$ & $545 \mathrm{HB}$ & $572 \mathrm{HB}$ & $600 \mathrm{HB}$ \\
\hline
\end{tabular}

\subsection{Otimização por Algoritmo Genético (AG) - Modelo Quadrático}

Para a aplicação do método Algoritmo Genético (AG), foi necessário imputar o modelo estatístico criado anteriormente e também as especificações referentes à dureza no software CrystalBall, que é um Software complementar ao software Excel®, versão 2010 e possui o recurso de otimização por Algoritmo Genético (AG). Foram realizadas cinco mil simulações em cada condição desejada e a análise referente à otimização por Algoritmos Genéticos (AG) será realizada posteriormente.

$\mathrm{Na}$ Tabela 5 (Planilha de programação para aplicação dos métodos de otimização), constam todas as informações necessárias para o desenvolvimento e a aplicação dos métodos de otimização (essas informações abasteceram o software CrystalBall). Assim como, as especificações, constantes atribuídas ao modelo estatístico, ajustes de níveis de fatores, predições geradas pelo modelo e os resultados alcançados (em porcentagem) para a propriedade mecânica dureza.

Tabela 5 - Planilha de programação para aplicação dos métodos de otimização.

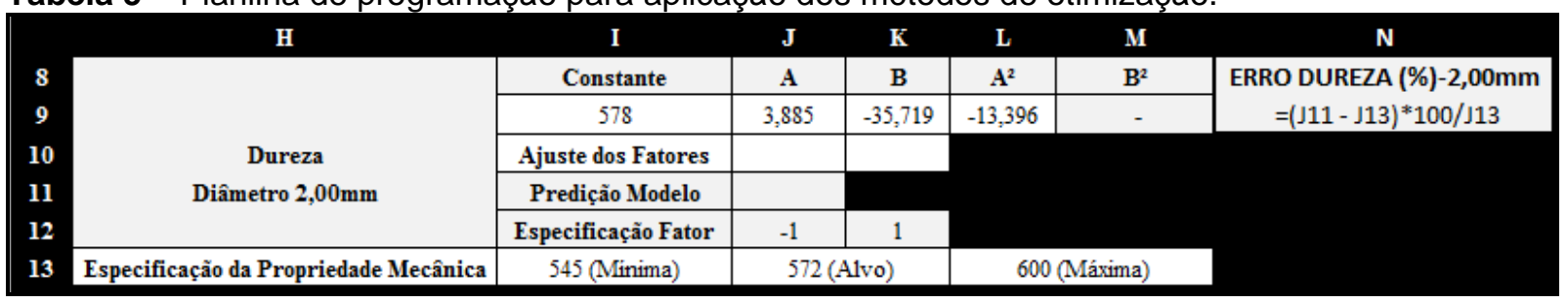

A descrição das informações necessárias para a aplicação dos métodos de otimização, constam a seguir:

a. Especificações: As especificações referentes à variável resposta dureza, constam nas células $I_{13}, J_{13}$ e $L_{13}$. Todavia, essas células são somente os 
locais de colocação dos dados, variando as especificações dos clientes, conforme forem sendo simuladas as situações.

b. Variáveis de decisão: Neste caso, refere-se aos valores de ajustes dos fatores A, e B, para que seja proporcionada a melhor condição de atendimento das especificações das respostas mecânicas.

c. Restrições: Algumas restrições foram imputadas para restringir os valores mínimos e máximos para os valores dos fatores $A$ e $B$ (células $J_{10}$ e $K_{10}$ ) foram restringidos a um mínimo e um máximo de -1 a 1 (correspondente à variável padronizada do estudo). Enquanto os valores das predições do modelo (célula: $\mathrm{J}_{11}$ ) foram restritas às suas especificações, em relação à propriedade mecânica vigente.

d. Função objetivo: Para a função objetivo foi utilizada a Distância Percentual Média (DPM), que é a média da distância das predições das variáveis respostas, ou seja, para cada resposta predita pelo modelo subtrai-se o valor "alvo"; então se obtém uma unidade de erro entre a predição e a especificação nominal, que deverá ser multiplicada por cem, para utilização em porcentagem (\%). Neste caso, a função objetivo será a minimização da média desses "erros", buscando assim uma condição que reduza ao máximo os valores das distâncias simultaneamente das duas variáveis respostas simultaneamente, buscando um equilíbrio entre o melhor ajuste das respostas. A função objetivo consta na célula No (Tabela 7).

\section{OTIMIZAÇÃO POR RECOZIMENTO SIMULADO (SIMULATED ANNELING)}

Nesta etapa, foi utilizada a Meta-heurística Recozimento Simulado (Simulated Anneling), com objetivo de encontrar o melhor ajuste possível dos fatores para otimização da variável respostas dureza.

O método Recozimento Simulado foi aplicado utilizando o software Scilab Enterprises (Software para computação numérica). Para programar o software 
(inserindo o modelo estatístico) e otimizar a variável resposta foi incorporada a programação contida na Tabela 6 .

Tabela 6 - Programação Scilab para modelagem estatística

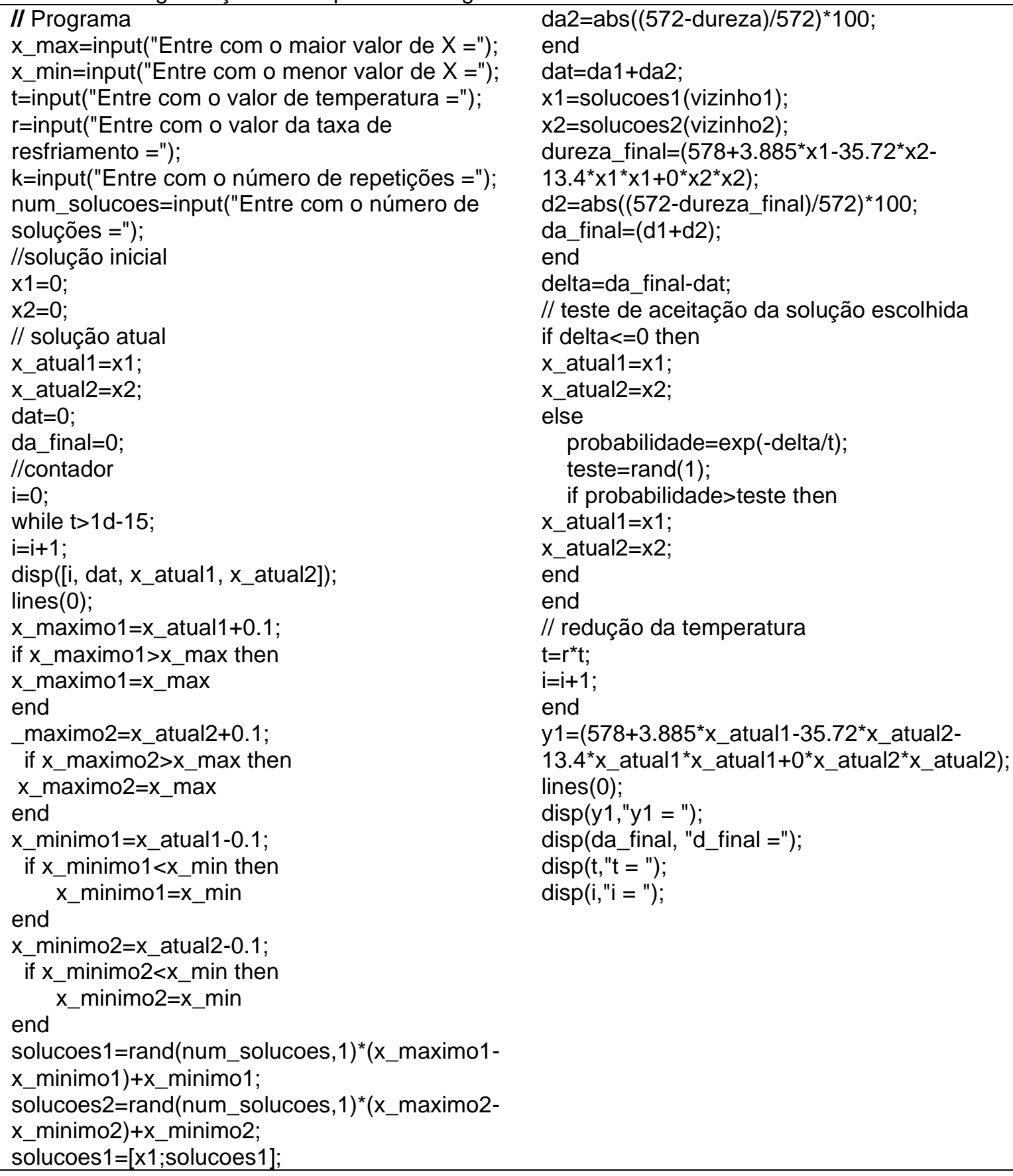


solucoes2 $=[\mathrm{x} 2$;solucoes2];

for $m=1: k$

//escolhe vizinho aleatoriamente

vizinho $1=$ int $32\left(\operatorname{rand}(1)^{*}(\right.$ num_solucoes -1$\left.)+1\right)$;

vizinho2 $=$ int32 $\left(\right.$ rand $(1)^{*}($ num_solucoes -1$\left.)+1\right)$;

dureza $=\left(578+3.885^{*} x \_\right.$atual $1-35.72^{*} x \_$atual $2-$

$13.4^{*} x \_$atual $1{ }^{*} x \_$atual $1+0^{*} x \_$atual2 ${ }^{*} x \_$atual 2$)$;

Foram realizadas 2245 simulações, até que fosse atingida a melhor condição de ajustes para obtenção da variável resposta e os resultados constam na Tabela 7 . As simulações foram apresentadas de forma resumida, sendo $\mathrm{y}_{1}$ os valor da propriedade mecânica dureza, sendo A (fator velocidade) e B (fator temperatura de chumbo).

Tabela 7- Recozimento Simulado (Scilab)- Modelagem quadrática
Entre com o maior valor de $X=1$
Entre com o menor valor de $X=-1$
Entre com o valor de temperatura $=1 \mathrm{~d} 10$
Entre com o valor da taxa de resfriamento $=0.95$
Entre com o número de repetições $=500$
Entre com o número de soluções $=100$

\begin{tabular}{lrc}
\hline 1. & Fator A. & Fator B \\
3. & -0.0085217 & 0.0478239 \\
5. & -0.0442730 & 0.1209136 \\
7. & 0.0459815 & 0.1071600 \\
2241. & -0.9923138 & -0.3241123 \\
2243. & -0.9923138 & -0.3241123 \\
2245. & -0.9923138 & -0.3241123 (melhor ajuste)
\end{tabular}

$\mathbf{Y}_{\mathbf{1}}=572.52735 \mathrm{HB}$

\section{AVALIAÇÃO dOS MÉTOdOS DE OTIMIZAÇÃO}

Após a constatação dos resultados obtidos, pela otimização da variável resposta dureza, por meio dos métodos Algoritmo Genético e da Meta-heurística Recozimento Simulado, serão realizadas simulações para os três principais clientes deste produto, para avaliar o desempenho desses métodos.

$\mathrm{Na}$ Tabela 8, constam as especificações dos clientes deste produto (referentes a dureza). Também foram atribuídos valores alvos para cada cliente e a 
predição alcançada em cada método de otimização, assim como, os ajustes dos níveis dos fatores para cada situação.

Tabela 8 - Comparação de resultados dos métodos de otimização.

\begin{tabular}{lccc} 
& $\begin{array}{c}\text { Cliente 1 } \\
\text { Dureza } \\
\text { (HB) }\end{array}$ & $\begin{array}{c}\text { Cliente 2 } \\
\text { Dureza } \\
\text { (HB) }\end{array}$ & $\begin{array}{c}\text { Cliente 3 } \\
\text { Dureza } \\
\text { (HB) }\end{array}$ \\
\hline $\begin{array}{l}\text { Predição: } \\
\text { (Recozimento }\end{array}$ & 573 & 555 & 590 \\
$\begin{array}{l}\text { Simulado) } \\
\text { Predição (AG) }\end{array}$ & 572 & 585 & 555 \\
$\begin{array}{l}\text { Especificação } \\
\text { (mínima) }\end{array}$ & 545 & 530 & 500 \\
$\begin{array}{l}\text { Especificação } \\
\text { (máxima) }\end{array}$ & 600 & 580 & 700 \\
$\begin{array}{l}\text { Especificação } \\
\text { (alvo) }\end{array}$ & 572 & 555 & 600 \\
$\begin{array}{l}\text { Ajuste Fator A } \\
\text { (Recozimento }\end{array}$ & $-0,99$ & 0,11 & 0,11 \\
$\begin{array}{l}\text { Simulado) } \\
\text { Ajuste Fator A (AG) }\end{array}$ & $-1,00$ & 0,20 & 0,12 \\
$\begin{array}{l}\text { Ajuste Fator B } \\
\text { (Recozimento }\end{array}$ & $-0,32$ & 0,65 & $-0,33$ \\
$\begin{array}{l}\text { Simulado) } \\
\text { Ajuste Fator B (AG) }\end{array}$ & $-0,33$ & 0,65 & $-0,33$ \\
\hline
\end{tabular}

Analisando a Tabela 9 é possível verificar os erros das predições do modelo estatístico após a utilização dos dois métodos. Após analisar os erros médios referentes a cada um dos métodos de otimização (apresentados na Figura 5), é possível concluir que o método mais eficaz de otimização para esse processo é a Meta-heurística Recozimento Simulado (Simulated Anneling), que obteve um erro médio de $0,56 \%$, o que para esse processo é considerado um erro muito baixo.

Tabela 9 - Comparação dos erros dos métodos de otimização

\begin{tabular}{lccc}
\hline & $\begin{array}{c}\text { Cliente 1 } \\
\text { Dureza } \\
\text { (HB) }\end{array}$ & $\begin{array}{c}\text { Cliente 2 } \\
\text { Dureza } \\
\text { (HB) }\end{array}$ & $\begin{array}{c}\text { Cliente 3 } \\
\text { Dureza } \\
\text { (HB) }\end{array}$ \\
\hline $\begin{array}{l}\text { Erro \% } \\
\text { (Recozimento }\end{array}$ & $0,08 \%$ & $0,00 \%$ & $1,60 \%$
\end{tabular}




\begin{tabular}{lccccc} 
Erro $\%(A G)$ & $0,00 \%$ & $5,41 \%$ & & $7,50 \%$ \\
$\begin{array}{l}\text { Erro Médio } \\
\text { (\%) }\end{array}$ Total & (Recozimento Simulado) & (AG) & \\
clientes & & $0,56 \%$ & & $4,30 \%$ & \\
\hline
\end{tabular}

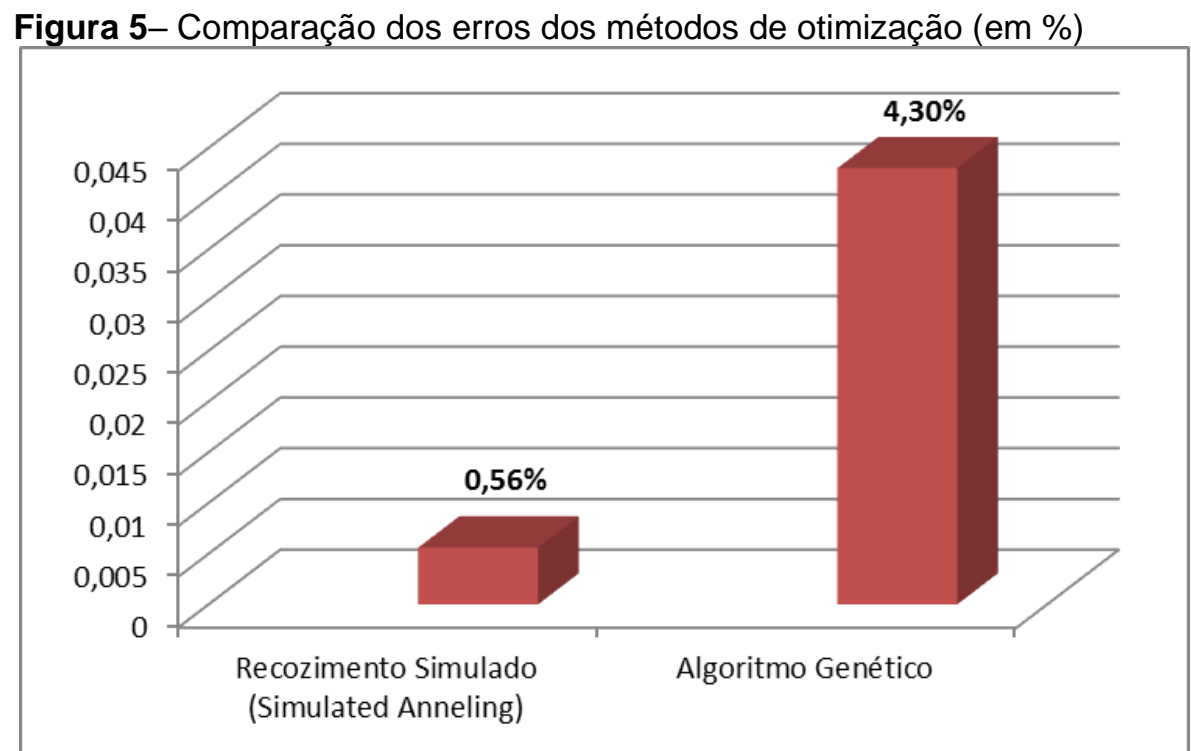

\section{CONCLUSÃO}

Observou-se que a melhor forma de modelagem deste processo é por modelagem quadrática, por meio da Metodologia de Superfícies de Resposta. Assim como, constatou-se que a melhor otimização para essa modelagem foi pela Metaheurística Recozimento Simulado (Simulated Anneling). No entanto, o método Algoritmo Genético (AG) não obteve um bom resultado para a modelagem estatística para esse processo. A justificativa da escolha da Meta-heurística Recozimento Simulado deu-se pelo menor índice médio de erro e também pela facilidade de alteração da programação de simulação do Software Scilab, pois com somente a alteração da "linha" de programação referente às especificações, uma nova simulação pode ser realizada. 
A implementação desta modelagem estatística, juntamente com as simulações realizadas utilizando o método de otimização escolhido, podem proporcionar a automatização dos fornos de têmpera, que por consequência poderia aumentar a produtividade deste processo, devido à redução do tempo de espera de resultados laboratoriais, menor amostragem para verificações, menor tempo de paradas de fornos devido à espera de resultados de ensaios mecânicos e também poderia contribuir para ganhos de qualidade, ocasionado por ajustes prédeterminados para melhor atendimento das especificações dos clientes.

O planejamento que antecedeu toda a fase experimental foi muito rigoroso, abordando detalhadamente os aspectos econômicos, tais como: investimentos necessários para a compra de matéria-prima (arame de aço trefilado), parada do processo produtivo para a execução dos experimentos (que representa turnos sem produção), manutenção dos fornos de têmpera e equipamentos de medição para prevenir falhas durante a experimentação e do envolvimento do departamento de Planejamento de Produção (PCP) para a correta organização da disponibilidade de insumos utilizados nos experimentos.

Outros custos também foram muito significativos, tais como: de energia elétrica, pois as fases de aquecimento dos fornos de têmpera utilizam energia elétrica para o seu funcionamento (e os custos com energia são relativamente altos), utilização do laboratório físico para os testes mecânicos (envolvendo todos os equipamentos e métodos para averiguação dos resultados), custos altos com funcionários para execução dos experimentos e para realização dos testes laboratoriais, custos com logística de movimentação de insumos e do produto acabado para os locais adequados e também com o salário do pesquisador que planejou toda sequencia experimental e analisou os dados.

Concluiu-se que os métodos estatísticos e de otimização estudados em ambiente acadêmico, muitas vezes empregados somente em pequena escala, em laboratórios de pesquisa e/ou simuladores nas universidades, se aplicam perfeitamente em ambiente industrial (chão de fábrica), resolvendo problemas complexos e sendo de grande valor para futuras inovações nas indústrias. Porém, a organização em ambiente industrial requer muito trabalho na fase de planejamento e na escolha dos métodos adequados que serão empregados durante e após a experimentação, pois um erro poderá significar a impossibilidade de realização do 
projeto definitivamente, visto que são empregados altos recursos financeiros em todas as etapas de realização e isso afeta diretamente os departamentos envolvidos, pois esses deverão interagir desde o planejamento e execução, até a análise dos dados.

\section{REFERÊNCIAS}

AJIC, D.; JOSIC, S.; PODRUG. Design of experiment's application in the optimization of milling process. Metalurgija, Sonja Josic, v.49, n.1, p. 123-126, oct. 2010.

BARROS, A. D.; MOCELLIN, J. V. Análise da flutuação do gargalo em flow shop permutacional com tempos de setup assimétricos e dependentes da seqüência. Gestão \& Produção, São Paulo, v. 11, n. 1, p. 101-108, jan. 2004. https://doi.org/10.1590/S0104$\underline{530 \times 2004000100009}$

BUZZO, W. R.; MOCELLIN, J. V. Programação da produção em sistemas flow shop utilizando um método heurístico híbrido algoritmo genético simulated annealing. Gestão \& Produção, São Carlos, v.7, n. 3, p. 364-377, dez. 2000. https://doi.org/10.1590/S0104$\underline{530 \times 2000000300012}$

BUZZO, W. R.; MOCELLIN, J. V. A influência da temperatura inicial no desempenho de um método híbrido algoritmo genético - Simulated annealing para a programação flow shop permutacional. 2005. 19 f. Dissertação (Mestrado em Engenharia de Produção)Escola de Engenharia de São Carlos, Universidade de São Paulo, São Paulo, 2005.

CALLISTER JR., W. D.; RETHWISCH, D. G. Ciência e engenharia de materiais: uma introdução. 8. ed. New York: LTC, 2012. 724 p.

CHAVES, A. A.; BIAJOLI, F. L.; MINE, O. M.; SOUZA, M. J. F. Metaheurísticas híbridas para resolução do problema do caixeiro viajante com coleta de prêmios. Produção, São Paulo, v.17, n. 2, p. 263-272, oct. 2007. https://doi.org/10.1590/S0103-65132007000200004

CHERRUAULT, Y.; MORA, G. Optimisation Globale - theorie des courbes $\alpha$-denses. 1. ed. Paris: Economica, 2005. 170 p.

CHIAVERINI, V. Aços e ferros fundidos. 7. ed. São Paulo: Associação Brasileira de Metalurgia e Materiais, 2012. 600 p.

COSTA, C. B. B. Modelagem detalhada e otimização de processos de cristalização. 2006. 150 f. Tese (Doutorado em Engenharia Química) - Universidade Estadual de Campinas, Campinas, 2006.

CRUZ, G. M. Avaliação das variáveis de processamento de normalização e têmpera dos aços de classificação ASTM516-70N e USIAR360Q no forno de tratamento térmico 2 da Usiminas. 2003. 74 f. Dissertação (Mestrado em Engenharia Metalúrgica e de Minas) Universidade Federal de Minas Gerais, Belo Horizonte, 2003.

DIÓGENES, A. N. Reconstrucção Tridimensional de Meios Porosos utilizando técnicas de Simulated Annealing. 2009. 147 f. Tese (Doutorado em Engenharia Mecânica) Universidade Federal de Santa Catarina. Florianópolis, 2009. 
FERREIRA, G.C.; SOUZA, M. J. F. S.; DUTRA, V.G. Metaheurísticas simulated annealing e iterated local search aplicadas ao problema de p-medianas capacitado: um estudo de caso. Journal of Materials Processing Technology, Sydney, v. 1, n. 1, p. 11-20, oct. 2008.

HOLLAND, J. H. Adaptation in natural and artificial systems. 1. ed. Cambridge: MIT press, $1975.500 \mathrm{p}$.

JUNIOR, H. A. O. Projeto de filtros digitais e separação de fontes usando fuzzy adaptive simulated annealing. 2008. $140 \mathrm{f}$. Tese (Doutorado em Engenharia)Universidade Federal do Rio de Janeiro. Rio de Janeiro, 2008.

KIRKPATRICK, S.; GELLAT, D. C.; VECCHI, M. P. Optimization by Simulated Annealing. Science, New York, v. 220, n. 1, p. 671-680, oct. 1983.

https://doi.org/10.1126/science.220.4598.671

LIBRANTZ, A. F. H.; ARAúJO, S. A. ; SANTANA, J. C. C.; BENVENGA, M. A. C. Cinética e otimização do processo de secagem do malte de milho usando simulated annealing. In: ENCONTRO NACIONAL DE ENGENHARIA DE PRODUÇÃO, 5., 2010. [Anais...]. São Carlos- Brasil, 2010. 1-17 p.

MARK, J. A.; SHARI, L. K. Using Design of Experiments. Paint \&Coatings Industry, Troy, v. 19 , n. 1 , p. $52-57$, oct. 2003.

MONTGOMERY, C. D. Design and analysis of experiments. 8. ed. New York: John Wiley \& Sons, 2013. 203 p.

MONTGOMERY, D. C.; RUNGER, G. C. Estatística aplicada e probabilidade para engenheiros. 2. ed. New York: LTC, 2003. 463 p.

NARA, K. Simulated annealing applications. Modern Optimization Techniques in Power Systems, Washington, v. 1, n. 1, p. 15-38, jan. 1999. https://doi.org/10.1007/978-94-015-9189$\underline{82}$

NETO, B. B.; SCARMINIO, I. S.; BRUNS, R. E. Como fazer experimentos: pesquisa e desenvolvimento na ciência e na indústria. 3. ed. Campinas: Unicamp, 2007. 480 p.

OLIVEIRA, S. A. Metaheurísticas aplicadas ao planejamento da expansão da transmissão de energia elétrica em ambiente de processamento distribuído. 2004. 150 f. Tese (Doutorado em Engenharia)- Universidade de Campinas, Campinas, 2004.

PAIVA, E. J. Otimização de manufatura com múltiplas respostas baseadas em índices de capacidade. 2006. 117 f. Dissertação (Mestrado em Engenharia)-Universidade Federal de Itajubá, Itajubá, 2006.

RODRIGUES; F. L.; LEITE, H. G., SANTOS, H. N.; SOUZA, A. L. Meta-heurística Algoritmo Genético para solução de problemas de planejamento florestal com restrições de integridade. Revista Árvore, São Paulo, v. 28, n. 2, p. 21-39, oct. 2004. https://doi.org/10.1590/S0100-67622004000200010

SILVA, K. G. Uso de simulated annealing e algoritmo genético no problema da reconfiguração de uma rede de distribuição de energia elétrica. 2013. $140 \mathrm{f}$.

Dissertação (Mestrado em Engenharia) - Universidade Federal do Rio Grande do Norte, Natal, 2013. 
SOUZA, M. J. F. Notas de aula da disciplina Inteligência Computacional para Otimização. Departamento de Computação, Instituto de Ciências Exatas e Biológicas, Universidade Federal de Ouro Preto, 2007.

TOFFOLO, T. A. M.; SOUZA, M. J. F. S.; SILVA, G.P. Resolução do problema de rodízio de tripulações de ônibus urbano via simulated annealing e iterated local search. 2005. 100 f. Monografia (Especialização em Computação) - Universidade Federal de Ouro Preto, Ouro Preto, 2005.

YAMAMOTO, L. Uso de simulated annealing e algoritmo genético no problema da reconfiguração de uma rede de distribuição de energia elétrica. 2004. $100 \mathrm{f}$.

Dissertação (Mestrado em Engenharia) - Universidade Federal do Paraná, Curitiba, 2004.

ZINI, E. O. C. Algoritmo Genético especializado na resolução de problemas com variáveis contínuas e altamente restritos. 2009. 100 f. Dissertação (Mestrado em Engenharia Elétrica) - Universidade Estadual Paulista, 2009.

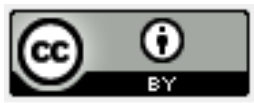

Artigo recebido em: 31/12/2020 e aceito para publicação em: 28/02/2021

DOI: http://dx.doi.org/10.14488/1676-1901.v21i1.4200 